\title{
Structural and magnetic studies on Co-Zn nanoferrite synthesized via sol-gel and combustion methods
}

\author{
B.B.V.S. VARA PRASAD ${ }^{1,2}$, K.V. RAMESH ${ }^{3, *}$, A. SRINIVAS ${ }^{4}$ \\ ${ }^{1}$ Department of Physics, MVGR College of Engineering, Chintalavalasa, Vizianagaram-535005, Andhra Pradesh, India \\ ${ }^{2}$ Department of Physics, JNTU Kakinada, Kakinada - 533003, Andhra Pradesh, India \\ ${ }^{3}$ Department of Electronics \& Physics, GITAM Institute of Science, GITAM Deemed to be University, \\ Visakhapatnam-530045, Andhra Pradesh, India \\ ${ }^{4}$ Defence Metallurgical Research Laboratory, Kanchanbagh, Hyderabad - 500058, Telangana, India
}

\begin{abstract}
Co-Zn nanocrystalline ferrites with chemical composition $\mathrm{Co}_{0.5} \mathrm{Zn}_{0.5} \mathrm{Fe}_{2} \mathrm{O}_{4}$ were synthesized by sol-gel and combustion methods. The sol-gel method was carried out in two ways, i.e. based on chelating agents PVA and PEG of high and low molecular weights. In auto-combustion method, the ratio of citric acid to metal nitrate was taken as 1:1, while in sol-gel method the chelating agents were taken based on oxygen balance. All the three samples were studied by thermogravimetric and differential thermal analysis for the identification of phase formation and ferritization temperature. The synthesized samples were characterized by powder X-ray diffraction and FT-IR spectroscopy without any thermal treatment. The measured lattice constants and observed characteristic IR absorption bands of the three samples are in good agreement with the reported values showing the formation of a cubic spinel structure. The crystallite sizes of all samples were determined using high intensity peaks and W-H plot. Size-Strain Plot method was also implemented since two of the samples showed low crystallite sizes. The least crystallite size $(5.5 \mathrm{~nm})$ was observed for the sample CZVP while the highest $(23.8 \mathrm{~nm})$ was observed for the sample CZCA. Cation distribution was proposed based on calculated and observed intensity ratios of selected planes from X ray diffraction data. All structural parameters were presented using experimental lattice constant and oxygen positional parameter, and they correlated with FT-IR results. Magnetic measurements were carried out using vibrating sample magnetometer at room temperature to obtain the characteristic parameters such as saturation magnetization, coercivity, remanence, squareness ratio and Bohr magnetons. Among all, the sample synthesized via citric acid autocombustion method displayed a remarkably higher magnetization of $53 \mathrm{emu} / \mathrm{g}$ and the remaining two samples displayed low magnetization values owing to their smaller crystallite sizes.
\end{abstract}

Keywords: nanoferrite; cation distribution; FT-IR spectroscopy; magnetization

\section{Introduction}

Cubic spinel ferrites are widely used in microelectronics, dynamic random access memories (DRAMs), high density data storage, high frequency applications and electromagnetic (EMI) suppressors. The chemical stability, high purity, excellent magnetic properties and high electrical resistivity made them ideal materials for the applications mentioned above.

Ferrites are also treated as mixed metallic oxides; they are ceramic magnetic materials with the general formula $\mathrm{AB}_{2} \mathrm{O}_{4}$, where $\mathrm{A}$ and $\mathrm{B}$ represent

*E-mail: kvramesh11@gmail.com divalent and trivalent metal ions. Ferrites crystallize in cubic spinel structure, which is named after the mineral spinel, $\mathrm{MgAl}_{2} \mathrm{O}_{4}$. The spinel crystal structure is determined primarily by the oxygen ions. This structure simply consists of metal ions enclosed by closed packed oxygen network. The unit cell of a spinel lattice contains eight molecules of $\mathrm{AB}_{2} \mathrm{O}_{4}$ in which 32 oxygen ions form a cubic close packed structure leaving 96 available interstitial sites (sublattices). Out of these 96 interstitial sites, 64 are tetrahedral (A) and 32 are octahedral (B) and each site is surrounded by four and six oxygen ions, respectively. These sites are only partially occupied by 24 metal ions. Based on the cation site preference, ferrites are 
classified into three classes: normal, inverse and mixed ferrites. In a normal spinel structure, the tetrahedral sites are occupied only by divalent ions and the octahedral sites are occupied by trivalent ions only. The distribution of ions in the spinel lattice is represented by the general formula $\left(\mathrm{A}^{2+}\right)\left[\mathrm{B}_{2}{ }^{3+}\right] \mathrm{O}_{4}{ }^{2-}$. The open parenthesis ( ) encloses ions on A-sites and the square brackets [ ] enclose ions on B-sites. Examples of such ferrites are zinc ferrite $(\mathrm{Zn})\left[\mathrm{Fe}_{2}\right] \mathrm{O}_{4}$ and cadmium ferrite $(\mathrm{Cd})\left[\mathrm{Fe}_{2}\right] \mathrm{O}_{4}$. In case of an inverse spinel structure, half the trivalent ions occupy tetrahedral sites; the octahedral sites are filled by the divalent ions and the remaining half of the trivalent ions. The distribution is represented by the general formula.Magnetite $\left(\mathrm{Fe}^{3+}\right)\left[\mathrm{Fe}^{2+} \mathrm{Fe}^{3+}\right] \mathrm{O}_{4}$ and nickel ferrite $\left(\mathrm{Fe}^{3+}\right)\left[\mathrm{Ni}^{2+} \mathrm{Fe}^{3+}\right] \mathrm{O}_{4}$ fall into this category. For mixed ferrites, both A- and B-sites are occupied both by divalent and trivalent ions. The distribution in this case is represented by the general formula $\left(\mathrm{Ax}_{\mathrm{x}}{ }^{2+} \mathrm{B}_{1-\mathrm{X}}{ }^{3+}\right)\left[\mathrm{A}_{1-\mathrm{X}^{2+}} \mathrm{B}_{1+\mathrm{X}}{ }^{3+}\right] \mathrm{O}_{4}{ }^{2-}$. Zinc-manganese, nickel-zinc and magnesiummanganese ferrites are examples of such structures.

In comparison with the bulk counterparts, nanoferrites show versatile, improved and more desirable structural, electrical and magnetic properties. Most of the recent works [1-4] on nanoferrites are focused on improvement of magnetic properties, such as magnetization, resistivity, reduction in particle size and controlled coercivity. The properties of ferrites change with the method of preparation. There are several reports available on different synthesizing methods to obtain reduced crystallite sizes. Among them there are ceramic method, co-precipitation, sol-gel, combustion synthesis, hydrothermal, spray pyrolysis and microwave synthesis [5]. Each method has its own advantages and disadvantages. The principal objective of all these methods is to produce smaller crystallites with uniform size distribution and homogeneous particles without any traces of intermediate compounds used in the synthesis. Based on the literature, sol-gel/auto-combustion is treated as the most reliable for producing homogenous, size controlled and less time consuming method among all wet chemical methods. The methods are based on the nature of chelating agent used. The methods of sol-gel or auto-combustion can be categorized based on the fuel or chelating agent used in the reaction and the amount of heat produced during the reaction [6]. In recent times, most of the researchers used polymer chelating agents, such as PVA, PEG or PVA, which were eventually burnt to form the final product. In this case, the precursor consisting of metal nitrates with polymer is heated up to $80{ }^{\circ} \mathrm{C}$ to dissolve polymer in the solution to form a polymer metal complex with improved ability to bind cations in the solution by organic polymer network connected with coordinate and intermolecular bonds. As such, the particle size is generally below $25 \mathrm{~nm}$. In sol-gel method (combustion method), when citric acid, glycine or urea is used as a fuel, a gel of weekly interconnected metal complex forms. Eventually, they are burnt to produce the final product. This method is also regarded as solution combustion method. Common features of solution combustion synthesis processes are fast heating rates, high-temperature and short reaction times. These characteristics make this method attractive for the manufacture of technologically important metallic oxide materials with high quality at a relative low cost. Major factors influencing solgel/combustion synthesis are the rate of evaporation of water during gelation as well as heat generated during the reaction and rate of heat energy supplied to the precursor [7].

In the available mixed ferrites, cobalt ferrites with zinc substitution have been studied less frequently, as they have large coercivity and low density, which are considered to be disadvantageous properties in the application perspective. Many researchers worked on materials, such as $\mathrm{Co}_{1-\mathrm{X}} \mathrm{Zn}_{\mathrm{X}} \mathrm{Fe}_{2} \mathrm{O}_{4}$ and $\mathrm{Cox}_{\mathrm{X}} \mathrm{Zn}_{1-\mathrm{X}} \mathrm{Fe}_{2} \mathrm{O}_{4}$ systems synthesized by different routes [8-10]. Among different available compositions of these $\mathrm{Co}-\mathrm{Zn}$ ferrites, $\mathrm{Co}_{0.7} \mathrm{Zn}_{0.3} \mathrm{Fe}_{2} \mathrm{O}_{4}, \mathrm{Co}_{0.6} \mathrm{Zn}_{0.4} \mathrm{Fe}_{2} \mathrm{O}_{4}$ and $\mathrm{Co}_{0.5} \mathrm{Zn}_{0.5} \mathrm{Fe}_{2} \mathrm{O}_{4}$ are considered as showing desirable properties, such as high saturation magnetization, moderate coercivity and squareness of the loop. Out of these three compositions, $\mathrm{Co}_{0.5} \mathrm{Zn}_{0.5} \mathrm{Fe}_{2} \mathrm{O}_{4}$ shows moderately high magnetization and moderate coercivity values in the range with a decent squareness of the loop, which are ideal characteristics for data storage materials. 
Several researchers studied this composition with divalent/trivalent substituent [11-13]. However, there are no reports on this composition synthesized individually by different synthesizing methods. The current research work is focused on investigating the composition of $\mathrm{Co}_{0.5} \mathrm{Zn}_{0.5} \mathrm{Fe}_{2} \mathrm{O}_{4}$ synthesized in sol-gel process with different chelating agents showing the best results for further dopant study. Phase purity, optimal crystallite size with enhanced magnetization and moderate coercivity are the main research parameters of this research work.

\section{Experimental}

\subsection{Synthesis}

The present composition $\mathrm{Co}_{0.5} \mathrm{Zn}_{0.5} \mathrm{Fe}_{2} \mathrm{O}_{4}$ was prepared by combustion and sol-gel methods using different chelating agents, namely citric acid, polyvinyl alcohol (PVA-high molecular weight) and polyethylene glycol (PEG-low molecular weight). High purity raw materials, cobalt nitrate Co $\left(\mathrm{NO}_{3}\right)_{2} \cdot 6 \mathrm{H}_{2} \mathrm{O}(99.5 \%)$, zinc nitrate $\mathrm{Zn}\left(\mathrm{NO}_{3}\right)_{2} \cdot 6 \mathrm{H}_{2} \mathrm{O}(99.5 \%)$, iron nitrate $\mathrm{Fe}$ $\left(\mathrm{NO}_{3}\right)_{3} \cdot 6 \mathrm{H}_{2} \mathrm{O}(99 \%)$ and citric acid $\left(\mathrm{C}_{6} \mathrm{H}_{8} \mathrm{O}_{7} \cdot \mathrm{H}_{2} \mathrm{O}\right)$ were taken in stoichiometric proportions and dissolved separately in minimum amount of deionized water. For sol-gel method, the amounts of PVA and PEG used in the reaction were taken using oxygen balance [6]. The formed metal hydroxide solutions were mixed thoroughly and stirred for an hour to improve the homogeneity. In the case of combustion method, an aqueous solution of citric acid in measured quantities (molar ratio of metal nitrates to citric acid as $1: 1$ ) was added slowly to the precursor containing metal nitrates dissolved in deionized water. This mixture was stirred about an hour until clear brownish solution occurred and the solution was heated up to around $65^{\circ} \mathrm{C}$ under continuous and constant stirring rate. The temperature was raised slowly up to $100{ }^{\circ} \mathrm{C}$ and then to $135^{\circ} \mathrm{C}$ and kept constant. Initially, the water was slowly evaporated and as the time proceeded, viscous liquid was formed leading to gelation. The internal temperature of the gel was slowly raised up to the stage where the gel started burning, resulting in formation of chunks of fluffy product and releasing of brownish gases. The entire reaction was completed in about $2 \mathrm{~h}$. After completion of the reaction, the flakes were grounded for $1 \mathrm{~h}$ (both wet and dry) using an agate mortar and a pestle to achieve a fine powder.

\subsection{Characterization techniques}

\section{Powder X-ray diffraction}

In order to confirm the structure of the output ferrite product and to make sure that the process adopted was correct, prior to further analysis, powder X-ray diffraction technique was employed to identify the structure of the material. Bruker D8 Advance Powder X-ray diffractometer with $\mathrm{CuK} \alpha$ $(\lambda=1.5406 \AA)$ radiation was used to characterize the samples.

\section{TGA/DTA analysis}

Thermogravimetric analysis (TGA) provides information about weight changes of a sample during the reaction, whereas differential thermal analysis (DTA) measures changes in temperature between a sample and a reference so as to reveal the exothermic or endothermic nature of the reaction as a function of temperature or time. PerkinElmer model Diamond TG/DTA instrument was used to record TGA/DTA curves of the present samples in the temperature range of $15{ }^{\circ} \mathrm{C}$ to $900{ }^{\circ} \mathrm{C}$ with an accuracy of $< \pm 0.5^{\circ} \mathrm{C}$.

\section{FT-IR spectroscopy}

FT-IR measurements were recorded using Perkin Elmer FT-IR spectrometer; it has a resolution of $1 \mathrm{~cm}^{-1}$ and scan range of $700 \mathrm{~cm}^{-1}$ to $50 \mathrm{~cm}^{-1}$. All measurements were recorded at room temperature using $\mathrm{KBr}$ pellet.

\section{Vibrating sample magnetometer}

Magnetic measurements were performed using LAKESHORE 7400 vibrating sample magnetometer up to 10000 Oe at room temperature. Saturation magnetization, coercivity, remnant magnetization and other related parameters were obtained from the recorded M-H curves data.

For simplicity, the present series of samples have been named as CZCA (sample synthesized with citric acid), CZPV (sample synthesized with PVA) and CZEG (sample synthesized with PEG). 


\section{Results and discussion}

\subsection{Structural studies}

\section{(a) Thermo-gravimetric analysis}

All samples were studied for the mass loss and ignition temperature of the reaction by thermogravimetric analysis. TGA/DTA curves of all samples are shown in Fig. 1a to Fig. 1c.

The TGA/DTA graph of the sample CZCA shows three different mass losses in the studied temperature range. The mass loss in the first region of $100{ }^{\circ} \mathrm{C}$ to $150{ }^{\circ} \mathrm{C}$ is attributed to water loss from the precursor. The next mass loss is observed in the region of $200{ }^{\circ} \mathrm{C}$ to $250{ }^{\circ} \mathrm{C}$, which represents the decomposition temperature of citric acid [14]. Further, the DTA curve shows an increasing trend above this temperature and around the temperature where the reaction becomes exothermic. At a temperature $495{ }^{\circ} \mathrm{C}$, it indicates a sharp exothermic peak, which is characteristic of citric acid/auto-combustion method. It is considered as ferritization temperature. TGA/DTA graphs of the samples CZPV and CZEG show a large initial mass loss due to typical polymer nature, moreover, they both show a huge mass loss in the region of $300^{\circ} \mathrm{C}$ to $350{ }^{\circ} \mathrm{C}$, which is attributed to the decomposition temperatures of the respective polymers $[7,15]$. In both samples, there is a noticeable mass loss in the region of $400{ }^{\circ} \mathrm{C}$ to $450{ }^{\circ} \mathrm{C}$, which is related to ferritization. It is connected with the exothermic nature of the curve after passing $400{ }^{\circ} \mathrm{C}$. Beyond this temperature both samples show different characteristic features. In CZPV sample, there is a broad exothermic peak in the region around $600{ }^{\circ} \mathrm{C}$ to $650{ }^{\circ} \mathrm{C}$, while in CZEG, the same was recorded in the region of $550{ }^{\circ} \mathrm{C}$ to $600{ }^{\circ} \mathrm{C}$. In CZPV sample, after passing the broad exothermic peak, the mass loss remains almost constant, while CZEG shows continuous mass loss till the end. This is attributed to the molecular weights of the polymers, i.e. the PVA used for the synthesis has a molecular weight of more than 100000 , whilst PEG has a molecular weight of 4000 . As a whole, the three samples display mass losses at a temperature above $400{ }^{\circ} \mathrm{C}$, which is characteristic of solgel and auto-combustion methods. However, the exothermic peaks of the three DTA curves occur at different temperatures. The sample CZCA, synthesized by citric acid shows a sharp exothermic peak below $500{ }^{\circ} \mathrm{C}$, the remaining samples display broad exothermic peaks above $550{ }^{\circ} \mathrm{C}$, which clearly differentiates the auto-combustion method from sol-gel method.

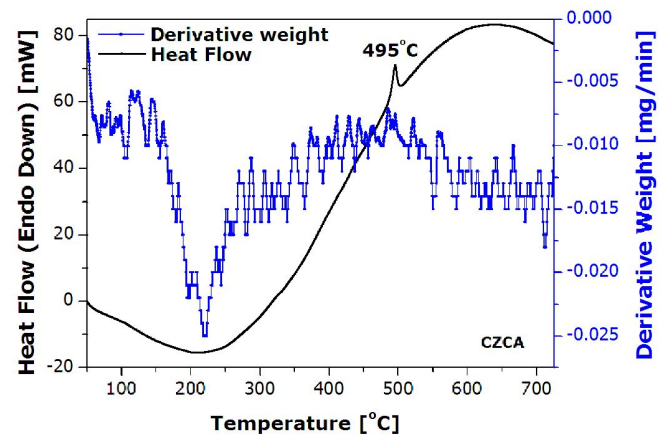

(a)

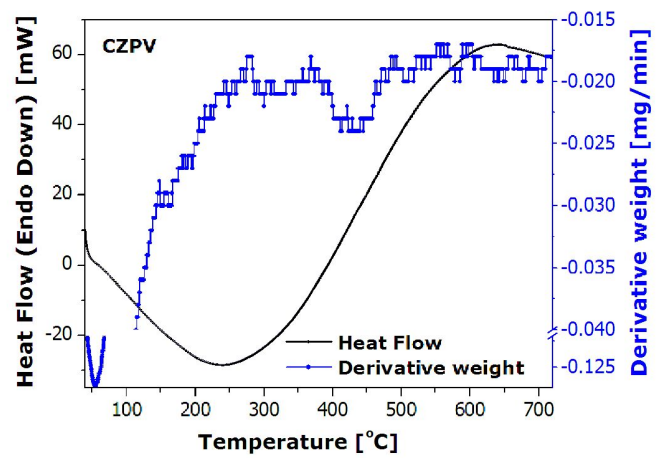

(b)

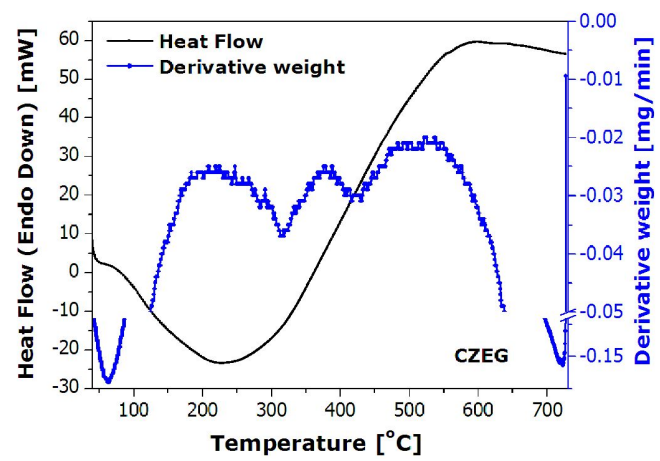

(c)

Fig. 1. TGA/DTA curves of a) CZCA, b) CZPV and c) CZEG samples.

\section{(b) Powder X-ray diffraction study}

The X-ray diffraction patterns of all ferrite samples confirmed the single-phase spinel structure. 
All peaks were indexed with known planes of cubic spinel, according the standard JCPDS cards of cobalt and zinc ferrites (JCPDS Card No. 89-1012, 22-1080, JCPDS Card No. 00-001-1121). From observation of the diffraction patterns, it is clear that the sample CZCA shows the highest peak intensities as well as resolved peaks especially in the case

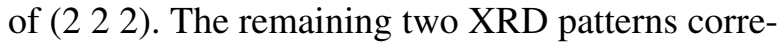
sponding to CZPV and CZEG display majority of the peaks but the peaks are not well resolved. All the peaks in these two patterns are very broad. This is related to size broadening and thus indicates the presence of fine crystallites. The powder X-ray patterns of the three samples are shown in Fig. 2.

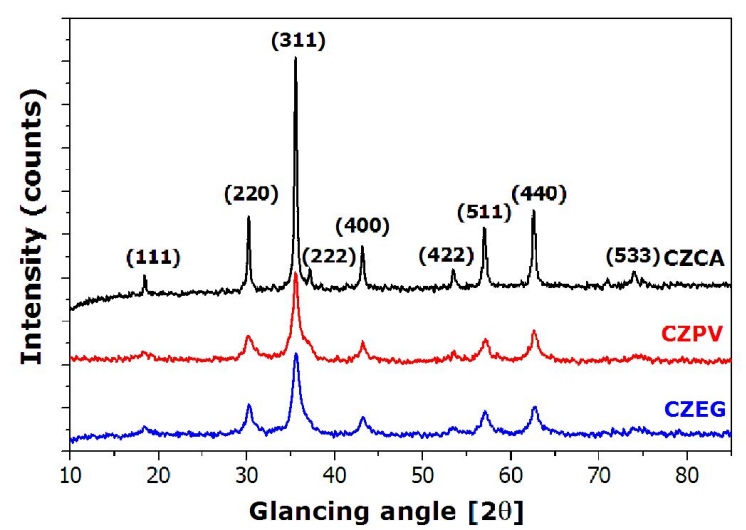

Fig. 2. Powder X-ray diffraction patterns of CZCA, CZPV and CZEG samples.

The interplanar spacing 'd' of different crystal planes has been obtained from Bragg law and the angles obtained for each peak from X-ray diffraction pattern,

$$
2 d_{h k l} \cdot \sin \theta=n \lambda
$$

Interplanar distances calculated from the glancing angle for all samples are presented in Table 1 and they are in agreement with the reported values except for CZPV and CZEG, which displayed smaller values resulting from the smaller crystallite size. The plane indices ( $\mathrm{h} \mathrm{kl}$ ) have been assigned to each peak using the X-ray data of standard spinel structure. The lattice parameter a has been calculated from each peak using equation:

$$
a=d \sqrt{h^{2}+k^{2}+l^{2}}
$$

The so obtained lattice constant values were extrapolated with Nelson-Riley function:

$$
F(\theta)=\frac{1}{2}\left(\frac{\cos ^{2} \theta}{\sin \theta}+\frac{\cos ^{2} \theta}{\theta}\right)
$$

[16] for accurate determination of lattice parameter. Lattice constants corresponding to high angle planes were taken into account for Nelson-Riley plot. The lattice constant and interplanar spacing of different crystal planes for the three samples are shown in Table 1.

The lattice constant values of the same composition were varied with respect to the method of synthesis. The variation of lattice constant was related to the variations in crystallite sizes and migration of cations between the sites, which is addressed in the next section. The obtained lattice constant values are in a good agreement with the reported values, indicating the entering of all cations into the respective spinel lattices.

\subsection{Determination of average crystallite size}

The broadening of X-ray diffraction peaks of all samples indicates the presence of smaller crystallites. The crystallite size D of all as-prepared samples has been calculated from the experimentally obtained full width at half maximum (FWHM) of most intense ( $\left.\begin{array}{lll}3 & 1 & 1\end{array}\right)$ peak in X-ray diffraction pattern, using Scherrer equation [17]:

$$
D=k / \beta \cos \theta
$$

where $\mathrm{k}$ is the Scherrer or shape factor (The Lorentzian and Gaussian limits for the shape factors are 0.6366 and 0.9394 , respectively [18]), $\lambda$ is the wavelength of $\mathrm{X}$-ray $(\mathrm{CuK} \alpha$ radiation $=1.5406 \AA$ ), $\theta$ is the Bragg angle and $\beta=\beta_{\mathrm{e}}$ - $\beta_{\mathrm{o}}$ from Warren formula, where $\beta_{\mathrm{e}}$ is the full width at half maximum (FWHM) of the diffraction peak in radians and the $\beta_{\mathrm{o}}$ instrumental broadening. In general, the profile fits of diffraction peaks are observed to be Lorentzian, Gaussian or Voigtshaped. In the present series of simple ferrites, the shape of the profile fit for the most intense diffraction peak (3 11 1) was found to be Lorentzian. 
Table 1. Lattice constant and interplanar spacing values of CZCA, CZPV and CZEG samples.

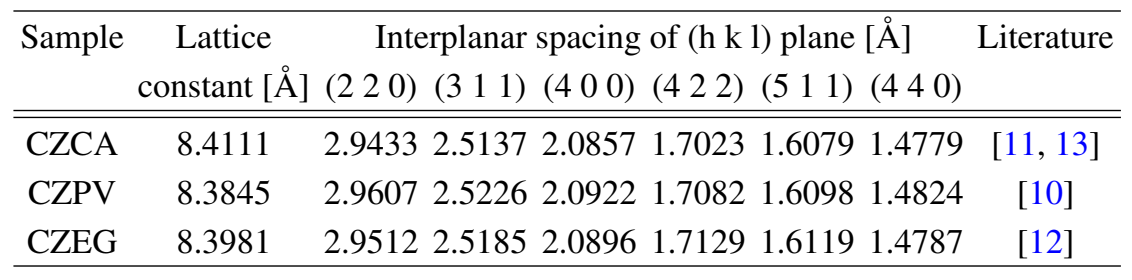

The accurate average crystallite size for as-prepared samples was determined using Williamson-Hall method. Accordingly, the broadening of X-ray diffraction peak is believed to be due to the crystallite size of the samples and the strain or lattice distortion. Mathematically it can be written as [18]

$$
\beta=\beta_{\text {size }}^{2}+\beta_{\text {strain }}^{2}
$$

In practice, this equation can be expressed as:

$$
\frac{\beta \cos \theta}{\lambda}
$$

where, $\beta$ is experimentally observed FWHM, $\beta_{\text {size }}$ is FWHM due to the effect of crystallite size only, $\beta_{\text {strain }}$ is FWHM due to the effect of microstrain only, D is crystallite size [nm], $\mathrm{k}$ is shape factor, $\epsilon$ is lattice strain, $\theta$ is incident angle of $\mathrm{X}$-ray and $\lambda$ is wavelength of X-ray beam.

Using this relation, contributions from crystallite size and microstrain can be separated in a straightforward approach by fitting the experimentally observed diffraction peak data. Separation of size and strain components can be done by plotting $\beta \cos \theta / \lambda$ versus $\sin \theta / \lambda$ (Williamson-Hall plots). If the resulting plot is a horizontal line, size broadening is the only significant contribution to peak width. If strain broadening is also important contribution, the plot is a linear function of $\sin \theta$. Williamson-Hall plots for all as-prepared ferrite compositions were drawn and the average crystallite size was calculated from the y-intercept of the plots by making strain component zero. The profiles of ( $\left.\begin{array}{lll}3 & 1 & 1\end{array}\right)$ peak after curve fitting along with W-H plots of all samples are shown in Fig. 3 a to $3 \mathrm{c}$. The crystallite size and the strain values are presented in Table 2.
Prior to W-H plot, FWHM values of most prominent peaks were calculated for all samples. Using these values, crystallite sizes were obtained and they were in the range of well below $29 \mathrm{~nm}$, which confirmed the formation nanocrystalline ferrites. The crystallite sizes of different peaks for each ferrite showed a little variation which is a sign of narrow distribution of particles. The crystallite sizes calculated from W-H plots were slightly smaller than those obtained from the (3 111 ) peak alone. This is a feature of $\mathrm{W}-\mathrm{H}$ plot, in which size broadening of a peak is only considered for crystallite size determination.

However, for calculating smaller crystallite sizes, W-H plot is not suitable since the linear coefficient value $\mathrm{R}$ for $\mathrm{W}-\mathrm{H}$ plot of CZCA was observed to be 0.94 whereas for the remaining two samples it was observed to be 0.47 and 0.13 . These low values of correlation coefficients of linear fits in W-H plots and negative slope values, especially for crystallites below $20 \mathrm{~nm}$, may be due to direct additive relation considered in W-H plot.

It may be eliminated by S-S plot (size-strain) method [18] in which, W-H plot is slightly modified as:

$$
\beta_{h k l}^{2}=\beta+\beta_{\text {strain }}^{2}
$$

Interplanar distance is a part of this relation and the complete equation can be expressed as:

$\left(d_{h k l} \beta_{h k l} \cos \theta\right)^{2} / \lambda^{2}=(k / D)\left(d_{h k l}^{2} \beta_{h k l} \cos \theta\right) / \lambda+$

Based on this relation, separation of crystallite of size and strain components can be done by plotting: $\left(d_{\mathrm{hk} 1} \beta_{\mathrm{hk} 1} \cos \theta\right)^{2} / \lambda^{2}$ versus $\left.\mathrm{d}_{\mathrm{h} \mathrm{k} 1}^{2} \beta_{h k_{l} l} \cos \theta\right) / \lambda$.

$\mathrm{S}-\mathrm{S}$ plots for all as-prepared ferrite compositions were drawn and the average crystallite size 
Table 2. Crystallite size and lattice strains of CZCA, CZPV and CZEG samples.

\begin{tabular}{cccccccc}
\hline Sample & \multicolumn{3}{c}{ Crystallite size [nm] } & \multicolumn{2}{c}{ Strain } & \multicolumn{2}{c}{ Correlation coefficient R } \\
& Scherrer & W-H plot S-S plot & W-H plot & S-S plot & W-H plot & S-S plot \\
\hline \hline CZCA & 28.3 & 27.8 & 23.8 & 0.00123 & 0.00138 & 0.9399 & 0.9968 \\
CZPV & 10.7 & 6.6 & 5.5 & -0.00088 & -0.0038 & 0.13 & 0.968 \\
CZEG & 9 & 7.2 & 7.4 & 0.00148 & 0.00267 & 0.4742 & 0.8986 \\
\hline
\end{tabular}

was calculated from the slope of the line, and microstrain was calculated from y-intercept of the plots. It is best suited for evaluating the smaller crystallite sizes of nanocrystalline materials. The correlation coefficients of the fit (R-values) are shown in Table 2. All R-values are very close to 1 showing the goodness of the fit even at narrow size distribution. Size-strain plots for the CZCA, CZPV and CZEG samples are shown in Fig. 4a to Fig. 4c. The crystallite sizes obtained by this method were observed to be slightly less than the values obtained by $\mathrm{W}-\mathrm{H}$ plot method. There is a slight difference in lattice strains of the three samples obtained from W-H plot and S-S plot. But, both have the same sign. The samples CZCA and CZEG show positive strain or, in other words, tensile strain, indicating the expansion of the lattice. In contrast, the sample CZPV shows a negative strain, an indication of compressive lattice strain, which is characteristic of a material of smaller crystallites.

Among all the samples, CZPV show the least crystallite size of $5.5 \mathrm{~nm}$ and CZCA have the highest crystallite size of $23.8 \mathrm{~nm}$. CZCA shows positive lattice strain indicating a small elastic expansion of the structure.

In general, the properties of a ferrite system vary noticeably depending upon the distribution of cations among tetrahedral and octahedral sites of the spinel lattice. The factors which can influence the distribution of the metal ions over the A- and B-sites are (1) their ionic radii and electronic configuration of the metal cations (2) crystal field stabilization energy or site preference energy (3) electrostatic energy of the spinel lattice and (4) particle size [16]. In the case of nanoferrites, particle size, chemical atmosphere and thermal energy (heat energy released during the reaction process) play a significant role in the cation distribution [6].
According the available literature reports [1113], cobalt has a strong octahedral preference, whereas zinc has a strong tetrahedral preference. However, in the present scenario, these site preferences cannot be taken into consideration as there was no additional thermal treatment and the crystallite size varied with the chelating agent. Consequently, it is convenient to check the intensities of two planes ( $\left.\begin{array}{lll}2 & 2 & 0\end{array}\right)$ and (4 40 ), which are sensitive to the cations on tetrahedral and octahedral, respectively [9]. The relative intensities of these two planes corresponding to the three samples with reference to the most intense plane (3 111 ) are shown in Table 3.

From Table 3, it is clear that the three samples show different deviations. This indicates that the cations in each sample are distributed differently. So, it was necessary to determine cation distribution for each sample separately. One of the most simple and reliable method to study the cation distribution is the calculation of intensities of X-ray diffraction peaks from the scattering power of the sites by varying the concentration of cations in either site. Cation distribution was proposed on the basis of intensity calculations and it was verified using theoretical lattice constant.

In order to evaluate theoretical intensities of the diffraction peaks, the following formula was used as suggested by Buerger [19]:

$$
I_{(h k l)}=\left|F_{h k l}\right|^{2} \cdot P \cdot L_{P}
$$

where $I_{h k l}$ is relative integrated intensity, $F_{h k l}$ is the structure factor, $\mathrm{P}$ is multiplicity factor and $\mathrm{L}_{\mathrm{P}}$ is the Lorentz polarization factor, which is expressed as:

$$
L_{P}=\left[\left(1+\cos ^{2} \theta\right) / \sin 2 \theta \cdot \cos \theta\right]
$$

The structure factor $F$ is a sum of scattering factors of constituent ions in that site. Atomic 
Table 3. Relative intensities of (2 20 ) and (4 40 ) planes of CZCA, CZPV and CZEG samples.

\begin{tabular}{cccc}
\hline \multirow{2}{*}{ Sample } & \multicolumn{3}{c}{ Relative intensity } \\
\cline { 2 - 4 } & $(220)$ & $(440)$ & difference \\
\hline \hline CZCA & 0.3030 & 0.2913 & 0.0117 \\
CZPV & 0.2053 & 0.1861 & 0.0192 \\
CZEG & 0.2259 & 0.2359 & -0.01 \\
\hline
\end{tabular}

scattering factor is defined as the ratio of amplitude of the incident wave scattered by all atoms to amplitude scattered by free electrons for the same incident beam. The intensities of diffracted beams from any crystal plane depend on the group of atoms in the unit cell and the scattering power of these atoms. In these set of theoretical X-ray diffraction intensities, Debye factor and the temperature factors were neglected since only the ratios of integrated intensities were considered. Furthermore, the orientations of crystal planes of the sample do not affect the ratios of intensities, which is allowed to consider intensity ratios rather than individual intensities [17].

Based on the relative intensities and their ability to influence cation arrangement in the spinel lattice, the crystal planes (2 20 ), (4 $\left.\begin{array}{lll}4 & 0\end{array}\right),\left(\begin{array}{lll}4 & 2 & 2\end{array}\right)$ and (4 40 ) were considered for intensity calculations. The crystal planes ( $\left(\begin{array}{lll}2 & 2 & 0\end{array}\right)$ and $\left(\begin{array}{lll}4 & 2 & 2\end{array}\right)$ are sensitive to any change of cations at A-site, while (4 40 ) is sensitive to the cations at octahedral site and (4 00 ) are sensitive to cations at both sites [16]. In entire range of the available diffraction intensities of different planes, ( $\left.\begin{array}{lll}3 & 1 & 1\end{array}\right)$ plane is nearly independent of the oxygen parameter and the cation distribution will not change significantly with such changes. Therefore, the intensity of ( $\left.\begin{array}{lll}3 & 1 & 1\end{array}\right)$ peak was taken as $100 \%$ [17]. All integrated intensities of the four planes under consideration were estimated with reference to $\left(\begin{array}{lll}3 & 1 & 1\end{array}\right)$ plane.

The structure factors of different crystal planes were calculated by using the following equations [16]:

$$
\begin{gathered}
F_{220}=8 F_{a} \\
F_{311}=2\left(4 F_{a}+4 \sqrt{ } 2 F_{b}\right) \\
F_{400}=\left(8 F_{a}-16 F_{b}-32 F_{o}\right)
\end{gathered}
$$

$$
\begin{gathered}
F_{440}=\left(8 F_{a}+16 F_{b}+32 F_{o}\right) \\
F_{422}=-8 F_{a}
\end{gathered}
$$

where $F_{A}, F_{B}$ and $F_{o}$ are structure factors of A-site, B-site cations and oxygen atoms.

All theoretical intensity ratios were estimated by continuous change of $5 \%$ of each divalent cation at either site. Based on theoretical intensity ratios, the cation distribution with the closest match with experimental values is presented in Table 4. Slight difference between calculated and observed intensities can be noticed. It is attributed to the atomic scattering factors considered for calculations derived from atoms at rest, i.e. at absolute zero. The X-ray diffraction patterns of all samples were recorded at room temperature. Although for all intensity ratios a minimum difference in theoretical and experimental ones is observed, the intensity ratio I(4 00 )/I( $\left.\begin{array}{lll}2 & 2 & 0\end{array}\right)$ shows the largest difference. The reason for this can be explained by the following considerations: (a) The presence of cation/anion vacancies in the system, which might be created in the process of nucleation and growth during the synthesis. This is a likely reason in nanoferrites. (b) Presence of cations in different oxidation states (for example $\mathrm{Fe}^{2+}$ in place of $\mathrm{Fe}^{3+}$ ). Furthermore, this method is based on the assumption that the specimens have random orientations in space (a version of ideally imperfect crystal). However, all polycrystalline specimens show more or less preferential orientation of the grains or in other words, a crystal may consist of some or all exactly parallel planes which may be nearly perfect and have low refracting power. This could be the reason for observed intensity differences in the specific planes. The proposed cation distribution is presented in Table 4. 
Table 4. Cation distribution and XRD plane intensity ratios of CZCA, CZPV and CZEG samples.

\begin{tabular}{|c|c|c|c|c|c|c|c|c|c|}
\hline \multirow{3}{*}{ Sample } & \multirow{3}{*}{ Cation distribution } & \multicolumn{8}{|c|}{ Intensity ratios } \\
\hline & & \multicolumn{4}{|c|}{ calculated } & \multicolumn{4}{|c|}{ observed } \\
\hline & & $(400) /(220)$ & $(400) /(422)$ & $(440) /(422)$ & $(440) /(220)$ & $(400) /(220)$ & $(400) /(422)$ & $(440) /(422)$ & $(440) /(220)$ \\
\hline CZCA & $\begin{array}{c}\left(\mathrm{Co}_{0.125} \mathrm{Zn}_{0.425} \mathrm{Fe}_{0.45}\right) \\
{\left[\mathrm{Co}_{0.375} \mathrm{Zn}_{0.075} \mathrm{Fe}_{1.55}\right] \mathrm{O}_{4}}\end{array}$ & 0.9053 & 0.8720 & 0.8186 & 0.8500 & 1.1892 & 0.8915 & 0.7208 & 0.9614 \\
\hline CZPV & $\begin{array}{c}\left(\mathrm{Co}_{0.175} \mathrm{Zn}_{0.45} \mathrm{Fe}_{0.375}\right) \\
{\left[\mathrm{Co}_{0.325} \mathrm{Zn}_{0.05} \mathrm{Fe}_{1.625}\right] \mathrm{O}_{4}}\end{array}$ & 0.9090 & 0.8747 & 0.8193 & 0.8515 & 1.0772 & 0.8744 & 0.7358 & 0.9064 \\
\hline CZEG & $\begin{array}{c}\left(\mathrm{Co}_{0.2} \mathrm{Zn}_{0.475} \mathrm{Fe}_{0.325}\right) \\
{\left[\mathrm{Co}_{0.3} \mathrm{Zn}_{0.025} \mathrm{Fe}_{1.675}\right] \mathrm{O}_{4}}\end{array}$ & 0.9101 & 0.8756 & 0.8197 & 0.8521 & 1.2419 & 0.8530 & 0.7174 & 1.0444 \\
\hline
\end{tabular}

Structural properties of spinel ferrites play a vital role in explaining the magnetic properties of these compounds such as magnetization, remanence etc. After obtaining a reliable cation distribution, all structural parameters were calculated and a detailed study was performed, which was then correlated with the data obtained from FT-IR spectrum.

The mean radii of the tetrahedral A- and octahedral B-sites are given by:

$$
\begin{aligned}
& r_{A}=x r_{M(A)}+y r_{M(A)}+(1-x-y) r_{F e(A)} \\
& r_{B}=(1 / 2)\left[(0.5-x) r_{M(B)}+(0.5-y) r_{M(B)}\right. \\
& \left.\quad+(1+x+y) r_{F e(B)}\right]
\end{aligned}
$$

where $\mathrm{r}_{M(A)}, \mathrm{r}_{M(B)}, \mathrm{r}_{F e(A)}$ and $\mathrm{r}_{F e(B)}$ are the radii of metal cations ( $\mathrm{Zn}$ and $\mathrm{Co}$ ) and iron ions at $\mathrm{A}$ and B-sites respectively (radii of cations at tetrahedral sites: zinc $(0.6 \AA)$, cobalt $(0.58 \AA)$, iron $(0.49 \AA)$ and for octahedral sites: zinc $(0.74 \AA)$, cobalt $(0.745 \AA)$, iron $(0.645 \AA)$ [20]).

The cation distribution in three samples with the same composition displayed different arrangement of cations over tetrahedral and octahedral sites. This clearly demonstrates that the cation distribution changes with crystallite size, method of preparation and the chelating agent (in the same synthesis). In the structure of spinel ferrites there are 24 interstitial sites filled by cations out of 96 available lattice sites; moreover, in nanoregime there is always a possibility of creation of cation/anion vacancies during the growth of crystalline compound at the completion of the reaction. Therefore, small variation in lattice constants may lead to greater deviations in the behavior of the same composition, as in the present case. The deviation of lattice results in either expansion or contraction of lattice, which is due to the movement of cations between the available sublattices. Since all the three samples showed positive/negative lattice strain, the presence of tensile strain/compressive strain is obvious. This indicates that there was a slight movement of anions (oxygen) surrounding the cations and this can be explained in terms of oxygen positional parameter ' $u$ '. Oxygen positional parameter can be presented by assuming the origin or center of symmetry either at A-site $(3 / 8,3 / 8,3 / 8)$ or at B-site $(1 / 4,1 / 4,1 / 4)$. Since A-site cation belongs to $\overline{4} 3 \mathrm{~m}$ symmetry group, the u parameter measured by considering origin at A-site, is denoted by $u^{43 m}$. Since the B-site cation belongs to $\overline{3} \mathrm{~m}$ symmetry group, the u parameter measured by considering origin at B-site, is denoted by $\mathrm{u}^{3 \mathrm{~m}}$ [21]. If the oxygen positional parameter $\mathrm{u}$ is measured by considering origin at A-site ( $\overline{4} 3 \mathrm{~m}$ symmetry group), it is represented as $\mathrm{u}^{43 \mathrm{~m}}$. The oxygen parameter can be calculated from known $r_{A}$ and $r_{B}$ values:

$$
u^{43 m}=0.3876\left(\frac{r_{A}}{r_{B}}\right)^{-0.07054}
$$

The choice of origin (the centre of symmetry) can be made by the relation:

$$
u^{43 m}=u^{3 m}+(1 / 8)
$$

The ideal values of $u^{43 m}=0.375, u^{3 m}=0.25$. The values of $\mathrm{u}^{43 \mathrm{~m}}, \mathrm{u}^{3 \mathrm{~m}}$ and theoretical lattice constants are shown in Table 4.

It is obvious from Table 4, that both $\mathrm{u}$ values are slightly larger than the ideal values, for which the 


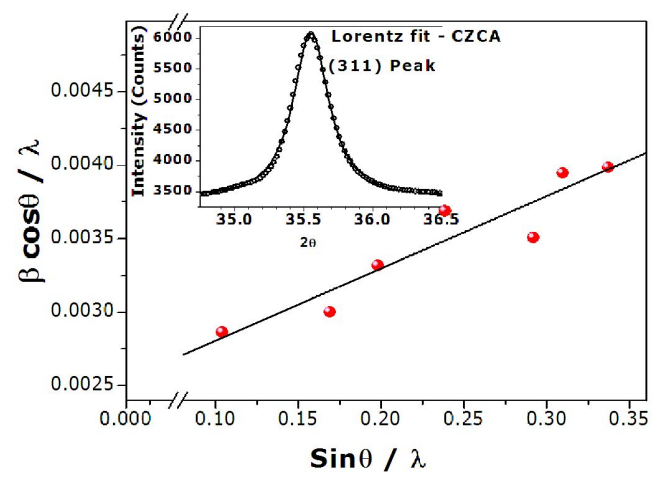

(a)

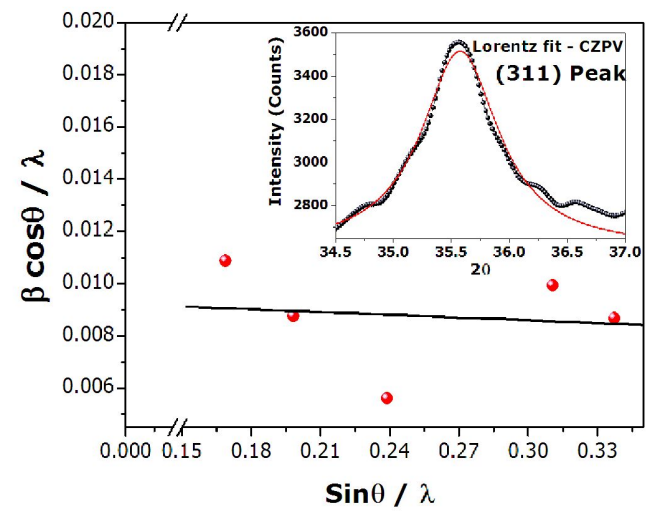

(b)

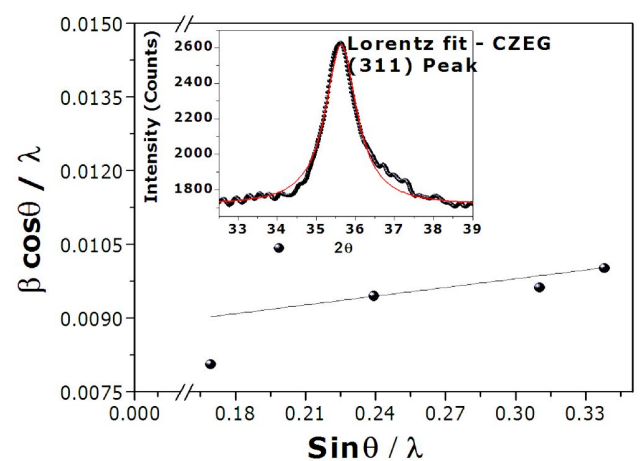

(c)

Fig. 3. Profile fits of ( $\left.\begin{array}{lll}3 & 1 & 1\end{array}\right)$ peak and Williamson-Hall plots of a) CZCA, b) CZPV and c) CZEG samples.

structure resembles inverse spinel structure. The mean tetrahedral radius is slightly higher in CZPV and CZEV samples, as the oxygen ions are moving away from tetrahedral coordinated cations along $\left\langle\begin{array}{lll}1 & 1 & 1\end{array}\right\rangle$ direction. This is also an indication of contraction of the octahedral sites and the inward

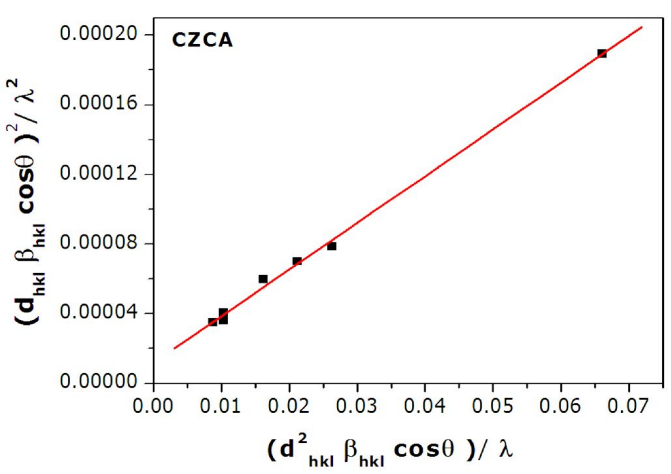

(a)

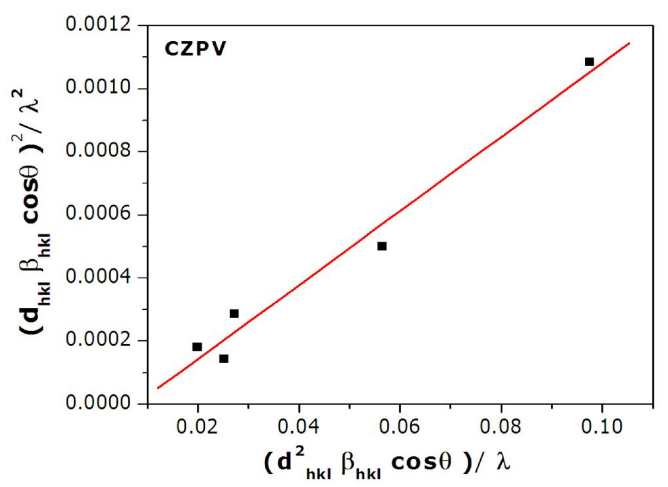

(b)

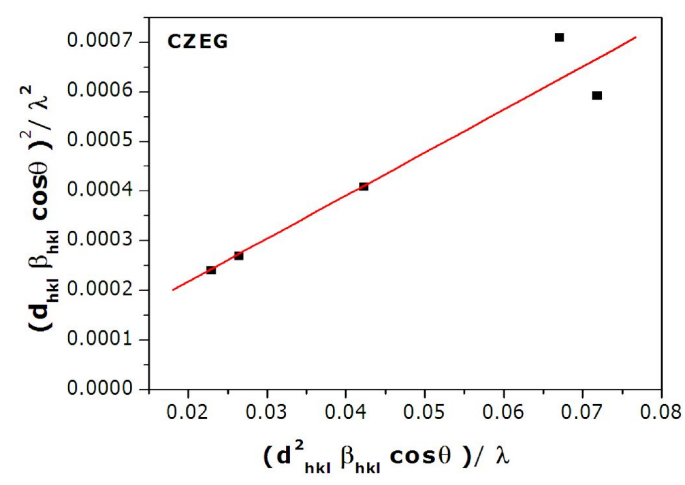

(c)

Fig. 4. Size-strain plots (S-S plots) of a) CZCA, b) CZPV and c) CZEG samples.

movement of oxygen ions along $\left\langle\begin{array}{llll}1 & 1 & 1\end{array}\right\rangle$ direction. Based on the cation distribution, it is evident that each sample has different concentrations of cations at both sites, along with the movement of $\mathrm{Fe}^{3+}$ ions, thus, it is quite possible to observe changes in structural parameters like bond lengths; shared and unshared edges, etc. 
The mean interionic distances (bond lengths) at tetrahedral $r_{t}$ and octahedral sites $r_{o}$ were estimated using the following relations [21]:

$$
\begin{gathered}
r_{t}=\sqrt{ } 3 a\left(u^{43 m}-(1 / 4)\right)-R_{o} \\
r_{o}=\left((5 / 8)-u^{43 m}\right) a-R_{o}
\end{gathered}
$$

The tetrahedral edge length $d_{\mathrm{AE}}$, the shared octahedral length $\mathrm{d}_{\mathrm{BE}}$, the unshared octahedral edge $\mathrm{d}_{\mathrm{BEu}}$, the inter-ionic distances (bond lengths) at tetrahedral $d_{A L}$ and octahedral sites $d_{B L}$ of the present nanoferrites have been calculated using the following equations and they are presented in Table 4:

$$
\begin{gathered}
d_{A E}=a \sqrt{ } 2\left(2 u^{43 m}-0.5\right) \\
d_{B E}=a \sqrt{ } 2\left(1-2 u^{43 m}\right) \\
d_{B E u}=a \sqrt{ }\left(4\left(u^{43 m}\right)^{2}-3 u^{43 m}+(11 / 16)\right. \\
d_{A L}=a \sqrt{ } 3(\delta+(1 / 8)) \\
d_{B L}=a \sqrt{ }\left(3 \delta^{2}-(\delta / 2)+(1 / 16)\right.
\end{gathered}
$$

where, $\delta$ represents the deviation from oxygen parameter, $\delta=\mathrm{u}^{3 \mathrm{~m}}-0.375$.

Based on the proposed cation distribution, the theoretical lattice constant [16] was calculated by using the relation:

$$
a_{t h}=[8 /(3 \sqrt{ } 3)]\left[\left(r_{A}+R_{o}\right)+\sqrt{ } 3\left(r_{B}+R_{o}\right)\right]
$$

where $R_{o}$ is the radius of oxygen ion (1.38 $\AA$ ).

The theoretical and experimental lattice constants are in agreement and the possible deviation can be explained on the basis of vacancy model [22].

The change of tetrahedral and octahedral symmetries due to anion dilation will not distort the unit cell dimensions, but it may slightly change the atomic positions. These, in turn, will reflect on bond lengths and bond angles. It is well known that the strength/magnitude of super-exchange interactions is directly proportional to bond angles and inversely proportional to bond lengths, thus, this parameter may provide useful information about the interactions present within the system. Cationcation (M-M) and cation-anion (M-O) bond distances as well as the angles between these interionic distances (bond angles) were estimated from the simplest trigonometric formulae available in the literature [21].

From Table 4, it is clear that all samples display the standard bond angles observed in ferrites. To recognize the deviations encountered in structural parameters in detail, vacancy model calculations were carried out to know the occupancy of ions in the lattice.

Ionic packing coefficients at tetrahedral and octahedral sites are:

$$
P_{A}=\frac{r_{t}}{r_{A}}, P_{B}=\frac{r_{o}}{r_{B}}
$$

Fulfillment coefficient:

$$
\alpha=\frac{32 \pi\left(r_{A}^{3}+2 r_{B}^{3}+4 R_{o}^{3}\right)}{3 V}
$$

where $r_{t}$ and $r_{o}$ are the interstitial radii and $r_{A}$ and $r_{B}$ are the average values of the ionic radii at the tetrahedral and octahedral sites, respectively, vacancy parameter $\beta=\frac{\left(a_{\mathrm{th}}^{3}-a_{\mathrm{exp}}^{3}\right)}{a_{\mathrm{th}}^{3}} \cdot 100 \%$.

The values of packing coefficients of $\mathrm{P}_{\mathrm{A}}$ and $P_{B}$ were nearly equal to 1 , which indicates very few possible cation/anion vacancies present in the samples. The same was displayed in the vacancy parameters whose values were in accordance with packing of ions. Vacancy model calculations indicated that all the samples showed values characteristic of nanoferrites: the vacancies which were slightly higher in CZPV and CZEG samples. The theoretical lattice constant was found to be larger than experimental value.

\section{(c) FT-IR studies}

The infrared spectra of all normal and inverse cubic spinel ferrites display four IR-active fundamentals $\mathrm{T}_{\mathrm{u}}$ in the vibrational spectra. Among 
Table 5. Values of structural parameters of CZCA, CZPV and CZEG samples.

\begin{tabular}{|c|c|c|c|c|}
\hline \multirow{2}{*}{ Parameter } & \multicolumn{3}{|c|}{ sample } & \multirow[t]{2}{*}{$\overline{\text { Literature }}$} \\
\hline & CZCA & CZPV & CZEG & \\
\hline Mean ionic radius at tetrahedral site $\mathrm{r}_{\mathrm{A}}[\AA]$ & 0.548 & 0.5553 & 0.5603 & \\
\hline Mean ionic radius at octahedral site $\mathrm{r}_{\mathrm{B}}[\AA]$ & 0.6673 & 0.6636 & 0.6612 & {$[8]$} \\
\hline Mean tetrahedral radius $r_{t}[\AA]$ & 0.5425 & 0.5408 & 0.5498 & \\
\hline Mean octahedral radius $r_{o}[\AA]$ & 0.6642 & 0.6552 & 0.6551 & \\
\hline Experimental lattice parameter (a) $[\AA]$ & 8.4111 & 8.3845 & 8.3981 & {$[9,12]$} \\
\hline Theoretical lattice parameter $\mathrm{a}_{\mathrm{th}}[\AA]$ & {$[\AA]$} & 8.4292 & 8.4304 & \\
\hline Oxygen parameter $\mathrm{u}^{3 \mathrm{~m}}$ & 0.382 & 0.3823 & 0.3827 & \\
\hline Oxygen parameter $\mathrm{u}^{43 \mathrm{~m}}$ & 0.257 & 0.2573 & 0.2577 & \\
\hline Shared tetrahedral edge $\mathrm{d}_{\mathrm{AE}}[\AA]$ & 3.1395 & 3.1366 & 3.1513 & {$[8,13]$} \\
\hline Shared octahedral edge $d_{\mathrm{BE}}[\AA]$ & 2.8080 & 2.7922 & 2.7871 & {$[8,13]$} \\
\hline Unshared octahedral edge $\mathrm{d}_{\mathrm{BEu}}[\AA]$ & 2.9761 & 2.9669 & 2.9720 & {$[8,13]$} \\
\hline Tetrahedral bond length $\mathrm{d}_{\mathrm{AL}}[\AA]$ & 1.9226 & 1.9207 & 1.9298 & \\
\hline Octahedral bond length $\mathrm{d}_{\mathrm{BL}}[\AA]$ & 2.0459 & 2.0371 & 2.0372 & {$[9,12]$} \\
\hline \multicolumn{5}{|l|}{ Cation-cation (M-M) bond distances [Å] } \\
\hline $\mathrm{b}$ & 2.9738 & 2.9644 & 2.9692 & \\
\hline $\mathrm{c}$ & 3.4871 & 3.4760 & 3.4817 & \\
\hline $\mathrm{d}$ & 3.6421 & 3.6306 & 3.6365 & \\
\hline $\mathrm{e}$ & 5.4632 & 5.4459 & 5.4547 & \\
\hline $\mathrm{f}$ & 5.1507 & 5.1344 & 5.1428 & \\
\hline \multicolumn{5}{|l|}{ Cation-anion (M-O) bond distances $[\AA]$} \\
\hline $\mathrm{p}$ & 2.0489 & 2.0467 & 2.0533 & \\
\hline $\mathrm{q}$ & 1.9058 & 1.8982 & 1.8921 & \\
\hline $\mathrm{r}$ & 3.6493 & 3.6348 & 3.6231 & \\
\hline s & 3.6569 & 3.6538 & 3.6513 & \\
\hline \multicolumn{5}{|l|}{ Bond angles [in degrees] } \\
\hline$\theta_{1}$ & 123.6656 & 123.5209 & 123.8277 & \\
\hline$\theta_{2}$ & 145.5400 & 145.4725 & 146.5235 & \\
\hline$\theta_{3}$ & 93.0516 & 92.8043 & 92.6076 & \\
\hline$\theta_{4}$ & 126.7148 & 126.1540 & 126.4068 & \\
\hline$\theta_{5}$ & 74.6387 & 74.7335 & 75.2829 & \\
\hline \multicolumn{5}{|l|}{ Vacancy model parameters } \\
\hline Packing coefficient: tetrahedral A-site $\left(\mathrm{P}_{\mathrm{A}}\right)$ & 0.9901 & 0.9739 & 0.9813 & \\
\hline Packing coefficient: octahedral B-site $\left(\mathrm{P}_{\mathrm{B}}\right)$ & 0.9953 & 0.9874 & 0.9908 & \\
\hline Fulfillment factor $(\alpha)$ & 0.6344 & 0.6403 & 0.6371 & \\
\hline Vacancy parameter $(\beta)$ & 0.5951 & 1.5851 & 1.1442 & \\
\hline
\end{tabular}

those four bands, the two high-frequency bands are broad and asymmetric, which strongly depends on the preparation conditions [23]. These two $v_{1}$ and $v_{2}$ bands are characteristic of lattice vibrations of oxide ions against metal ions in the lattice and are commonly observed in most of metallic oxides. The frequencies $v_{1}$ and $v_{2}$ correspond to tetrahedral and octahedral metal complexes, respectively. Two low-frequency bands $\left(v_{3}\right.$ and $v_{4}$ ) depend on the nature of divalent metal cation as well as the mass of the cation and they are unaffected by the preparation conditions [24]. In certain mixed ferrites, the presence of divalent metal ions at larger concentrations gives rise 
to structural changes within the lattice environment without affecting the spinel structure of the ferrite as a whole. Such structural changes brought about by metal ions migrating among the available tetrahedral and octahedral sublattices, which may be either lighter or heavier than the replaced divalent ion in the particular sublattice of ferrite, strongly influence the lattice vibrations. The FT-IR spectral study is considered to be important, sufficient and effective tool in the investigation of structural properties of mixed nanoferrites, because the four characteristic bands occur below $600 \mathrm{~cm}^{-1}$ and the far infrared studies are efficient in the range of $50 \mathrm{~cm}^{-1}$ to $700 \mathrm{~cm}^{-1}$.

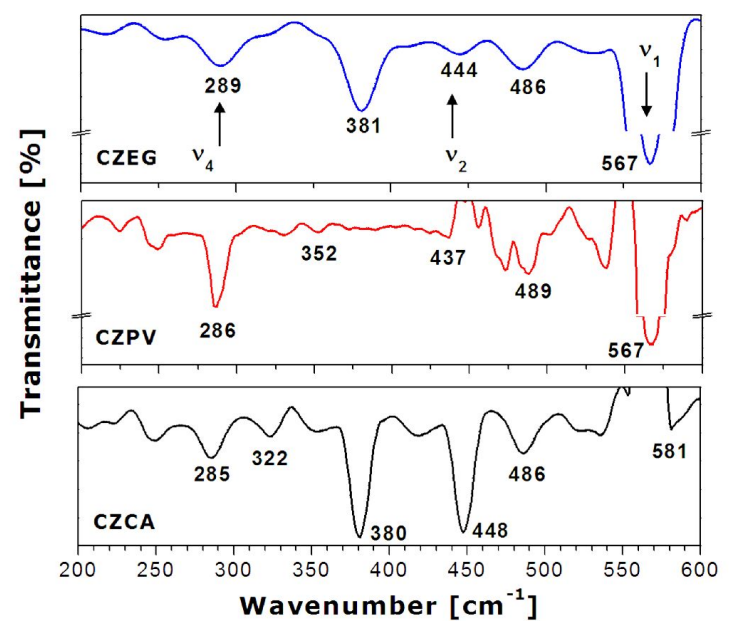

Fig. 5. FT-IR spectra of CZCA, CZPV and CZEG samples.

FT-IR spectra of all samples are shown in Fig. 5. All samples show characteristic absorption bands, accompanied by shoulders about each band. Among the two high frequency absorption bands, first absorption band of all samples is observed in the region of $567 \mathrm{~cm}^{-1}$ to $581 \mathrm{~cm}^{-1}$, CZCA shows a band at $581 \mathrm{~cm}^{-1}$, whereas the remaining two samples show the same peak at $567 \mathrm{~cm}^{-1}$. The absorption band depth recorded for CZPV and CZEG is quite high compared to the variation of iron ions in each sample, since $v_{1}$ corresponds to $\mathrm{Fe}^{3+}-\mathrm{O}$ bonds at tetrahedral sites. Second absorption band of all samples is observed in the region of $444 \mathrm{~cm}^{-1}$ to $448 \mathrm{~cm}^{-1}$ and absorption band depth recorded for CZPV and CZEG is quite low compared to the variation of iron ions in each sample, since $v_{2}$ corresponds to $\mathrm{Fe}^{3+}-\mathrm{O}$ bonds at octahedral sites. This is quite opposite to what happened in case of $v_{1}$. As such, it supports the cationic distribution derived from $\mathrm{X}$ ray diffraction in which iron concentration in tetrahedral and octahedral sites varied with each sample. The third band $v_{3}$ occurs in the range of $352 \mathrm{~cm}^{-1}$ to $381 \mathrm{~cm}^{-1}$, which corresponds to divalent cations at octahedral sites. It is one of characteristic bands in mixed ferrites [23, 24]. The fourth band $v_{4}$ is observed in the range of $285 \mathrm{~cm}^{-1}$ to $289 \mathrm{~cm}^{-1}$, which is assigned to the vibrations of divalent ions at tetrahedral sites. The positions of the all characteristic bands for all samples are shown in Table 6.

Table 6. Characteristic absorption peaks and force constants of bonds in tetrahedral and octahedral metal complexes of CZCA, CZPV and CZEG samples.

\begin{tabular}{|c|c|c|c|c|c|}
\hline \multirow[t]{2}{*}{ Sample } & \multicolumn{4}{|c|}{$\begin{array}{c}\text { Position of the } \\
\text { absorption band }\left[\mathrm{cm}^{-1}\right.\end{array}$} & \multirow[t]{2}{*}{ Literature } \\
\hline & $v_{1}$ & $v_{2}$ & $v_{3}$ & $v_{4}$ & \\
\hline CZCA & 581 & 448 & 380 & 285 & {$[8,11]$} \\
\hline CZPV & 567 & 437 & 352 & 286 & - \\
\hline CZEG & 567 & 444 & 381 & 289 & [10] \\
\hline
\end{tabular}

\subsection{Magnetic measurements}

The M-H loop measurements were taken in the range of 0 Oe to $10000 \mathrm{Oe}$ at room temperature. The M-H loops of three samples synthesized in different routes are presented in Fig. 6.

In general, magnetization of ferrites can be explained on the basis of Neel two sublattice model according to which, a ferrite material possesses collinear ferromagnetic structure. This structure consists of two sublattices aligned antiparallel to each other in such a manner that magnetic moment of one sublattice is always trying to cancel out the other. Since the density of magnetic ions is always high in octahedral sites, so the net magnetic moment is considered as the difference between magnetization of octahedral and tetrahedral sites [25]. This model is applicable to samples having 
relatively good crystalline structure. In present samples, CZCA displays the highest magnetization of $53 \mathrm{emu} / \mathrm{g}$. This high magnetization is attributed to the strong ferromagnetic nature of cobalt ion occupying B-sites. From cation distribution, it is evident that majority of the cobalt ions are present at octahedral sites while fewer amount of cobalt occupies A-sites. Moreover, some of the nonmagnetic zinc ions occupy B-sites also. Owing to this, some of iron ions migrate to A-sites and thus increase the number of magnetic ions at A-sites. Due to this, there is a possibility of strong magnetic super exchange interactions between A- and B-sites, leading to higher magnetization.

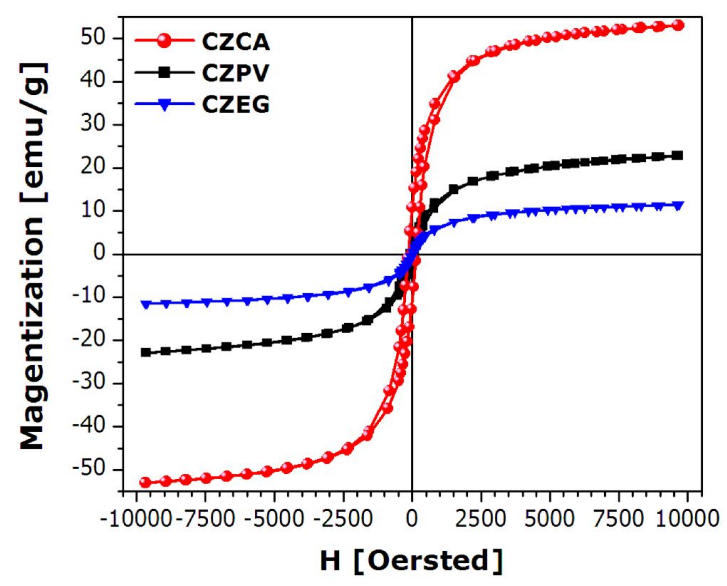

Fig. 6. M-H loops of CZCA, CZPV and CZEG samples at room temperature.

The magnetization of the remaining samples cannot be explained by the Neel two sublattice model, as it is not suitable for nanoferrites having a crystallite size less than critical size $25 \mathrm{~nm}$. In addition to this fact, the presence of few amounts of nonmagnetic zinc ions at octahedral sites results in the canting effects and disturbs the antiparallel alignment of sublattices. As a consequence, the net magnetization decreases [8]. In this case, Yafet-Kittel three sublattices model is used to explain the changes in magnetization. According to this model, the presence of non-collinear spin arrangement in the present system, where the dominant inter-sublattice A-B super exchange interactions become weaker, which facilitates the intra-sublattice B-B super-exchange interaction to become stronger. This leads to the random spin canting on the B-site, disturbing the antiparallel arrangement of spins at B-sites with respect to spins at A-sites. Therefore, it may be convincingly concluded that the canted (non-collinear) spins resulted in a decrease of the experimental magnetic moment (thus the magnetization). The increased favor for triangular spin arrangements on B-sites arising due to the smaller crystallite sizes, indicates the presence of canted spin arrangements in the CZPV and CZEG samples.

Experimental Bohr magnetons per one formula unit were calculated from the following relation [25]:

$$
n_{B}=\left(M_{w t} \cdot M_{s}\right) / 5585
$$

where $M_{w t}$ is the molecular weight and $M_{s}$ is the saturation magnetization, respectively.

The difference between experimental and theoretical Bohr magnetons is also due the fact that theoretical Bohr magnetons were considered at absolute temperature, while the experimental ones were calculated at room temperature using magnetization data.

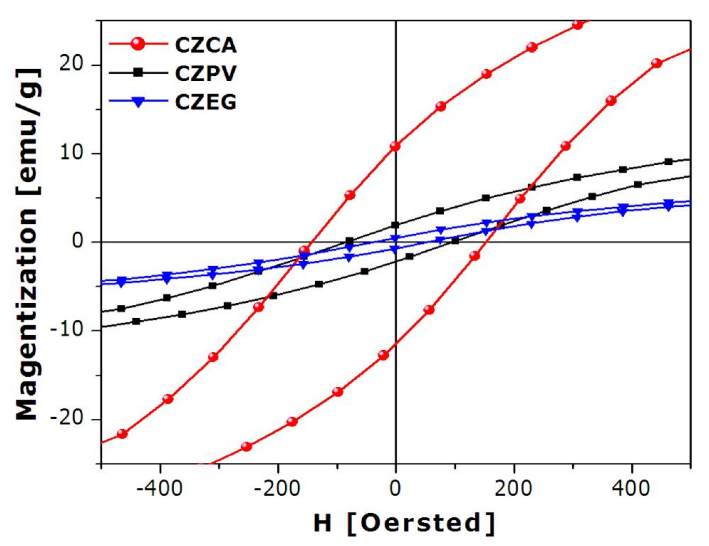

Fig. 7. Coercivity and remanence of CZCA, CZPV and CZEG samples.

The coercivity of all samples was due to the anisotropy of cobalt ions at octahedral sites. In particular, the CZCA samples showed the highest coercivity as a result of higher number of cobalt ions at B-sites and large crystallite size compared to the rest of the samples (Fig. 7). 
Table 7. Saturation magnetization, coercivity, remanence, squareness ratio, experimental and theoretical Bohr magnetons of CZCA, CZPV and CZEG samples.

\begin{tabular}{ccccccc}
\hline \multicolumn{2}{c}{$\begin{array}{c}\text { Sample Magnetization } \\
{[\mathrm{emu} / \mathrm{g}]}\end{array}$} & \multicolumn{3}{c}{ Bohr magnetons $[\mu \mathrm{B}]$} & \multicolumn{3}{c}{ Coercivity Remanence Squareness } \\
experimental theoretical & {$[\mathrm{Oe}]$} & {$[\mathrm{emu} / \mathrm{g}]$} & ratio \\
\hline \hline CZCA & 53 & 2.2572 & 6.25 & 150.75 & 11.8 & 0.22 \\
CZPV & 22.82 & 0.9719 & 6.7 & 92.5 & 2 & 0.088 \\
CZEG & 14.48 & 0.6167 & 7.05 & 47.5 & 0.65 & 0.045 \\
\hline
\end{tabular}

\section{Conclusions}

Nanocrystalline $\mathrm{Co}_{0.5} \mathrm{Zn}_{0.5} \mathrm{Fe}_{2} \mathrm{O}_{4}$ were synthesized by sol-gel and citric acid autocombustion methods. All three samples produced by these methods were composed of single phase nanocrystalline material as it was confirmed by powder $\mathrm{X}$-ray diffraction. The lattice constants of the nanoferrites were in good agreement with the reported values. Crystallite size calculated from $\mathrm{W}-\mathrm{H}$ plot, gave an average size of $27.8 \mathrm{~nm}$ with the best coefficient of fit for the CZCA sample. However, the remaining samples demonstrated very low coefficients due to smaller crystallite sizes. This problem was eliminated by S-S plot and accurate crystallite sizes were given with the best coefficient of fit. Cation distribution was assessed from XRD intensity ratios and in all ferrites divalent cations were found to occupy both tetrahedral and octahedral sites. Cation distribution differed greatly depending on the method of preparation and the crystallite size indicated that site preference of divalent cations changes significantly in nanoregime. FT-IR study displayed four characteristic absorption bands of the nanoferrites and the band positions showed that divalent cations are distributed over both sites. The vacancy model calculations indicated that there were possible cation/anion vacancies in the samples synthesized via sol-gel method, in which least crystallite sizes were observed. Furthermore, during the growth process and for extremely small crystallite size, some of the ions may fail to occupy the interstitial sites. The M-H loops of all three samples showed different magnetizations. The change in magnetization values for the same compositions was attributed to variations in superexchange interactions, crystallite size and spin canting effects. Among the three as-prepared samples, CZCA displayed high magnetization (53 emu/gm), moderate coercivity $(150 \mathrm{Oe})$ and squareness ratio close to 0.22 , which are essential characteristics for data storage materials. Crystallite sizes calculated from $\mathrm{W}-\mathrm{H}$ and S-S plots for this sample gave average values of 27.8 and $23.8 \mathrm{~nm}$, respectively, with best coefficients of fit.

\section{References}

[1] RaghVEndRA SINGH Y., JAROMIR H., Miroslav H., Pavol S., Cigan A., Martin P., Eva B., Martin B., FrantišKa F., Jiri M., Martin Z., Lukas K., Miroslava H., VojtĚch E., $J$. Magn. Magn. Mater, 378 (2015), 190.

[2] Dzmitry K., Maria I., Vladimir P., Yulia F., Solid State Sci., 39 (2015), 69.

[3] Majid Niaz A., Rahman A., Sulong A.B., Muhammad A.K., J. Magn. Magn. Mater, 421 (2017), 260.

[4] Manikandan V., Vanitha A., Ranjith KUMAR E., Kavita S., J. Magn. Magn. Mater, 426 (2017), 11.

[5] Tasawar J., Asghari M., Akhlaq A.M., J. Supercond. Nov. Magn., 24 (2011), 2137.

[6] Andris S., Gundars M., Front. Mater. Sci., 6 (2012), 128.

[7] Kashinath C.P., Aruna S.T., Tanu M., Curr. Opin. Solid St. M., 6 (2002), 507.

[8] Raut A.V., Barkule R.S., Shengule D.R., JaDHAV K.M., J. Magn. Magn. Mater., 358 - 359 (2014), 87.

[9] Deraz N.M., Alarifi A., J. Anal. Appl. Pyrol., 94 (2012), 41.

[10] Gozuak F., Koseoglu Y., Baykal A., Kavas H., J. Magn. Magn. Mater, 321 (2009), 2170.

[11] Shivaji R.K., SAnJaY S.K., Pramod N.J., VAShishtha M.G., DURyodhan P.W., Govind B.K., SANDIP R.S., SAMBhaJi R.B., Mater. Lett., 84 (2012), 169.

[12] Sonal S., Sharma R., Namgyal T., Jauhar S., Bhukal S., KaUR J., Ceram. Int., 38 (2012), 2773.

[13] Bhukal S., Mor S., Bansal S., Singh J., SingHAL S., J. Mol. Struct., 1071 (2014), 95. 
[14] Danks A.E., Hall S.R., Mater. Horiz., 3 (2016), 91.

[15] Seongok Han, Kim C., Kwon D., Polymer, 38 (1997), 317.

[16] Vara Prasad B.B.V.S., Mod. Phys. Lett. B, 28 (2014), 1450155.

[17] Lakhani V.K, Pathak T.K., Vasoya N.H., Modi K.B., Solid State Sci., 13 (2011), 539.

[18] Maniammal K., Madhu G., Bijua V., Physica E, 85 (2017), 214.

[19] BuERGER M.J., Crystal Structure Analysis, Wiley, New York, 1960.

[20] Shannon R.D., Acta Crystallogr. A, 32 (1976), 751.

[21] VARA PRASAD B.B.V.S., RAJESH BABU B., Sivaram Prasad M., Mater. Sci.-Poland, 33 (2015), 806.
[22] Mohammed K.A., Al-Rawas A.D., Gismelseed A.M., Sellai A., Physica B, 407 (2012), 795.

[23] Josyulu O.S., Sobhanadri J, Phys. Status Solidi A, 65 (1981), 479.

[24] Reddy P. V., Salagram M., Phys. Status Solidi A, 100 (1987), 639.

[25] Prasad M. S. R., Prasad B. B.V.S.V., Rajesh B., RAO K. H., RAMESH K.V., J. Magn. Magn. Mater, 323 (2011), 2115.

Received 2017-05-26

Accepted 2018-05-17 